\title{
Factorization of Functional Operators with Involutive Rotation on the Unit Circle
}

\author{
Aleksandr Karelin*, Anna Tarasenko \\ Institute of Basic Sciences and Engineering, Hidalgo State University, Pachuca, Mexico \\ Email: *karelin@uaeh.edu.mx, anataras@uaeh.edu.mx
}

How to cite this paper: Karelin, A. and Tarasenko, A. (2020) Factorization of Functional Operators with Involutive Rotation on the Unit Circle. Applied Mathematics, 11, 1132-1138.

https://doi.org/10.4236/am.2020.1111076

Received: October 12, 2020

Accepted: November 13, 2020

Published: November 16, 2020

Copyright $\odot 2020$ by author(s) and Scientific Research Publishing Inc. This work is licensed under the Creative Commons Attribution International License (CC BY 4.0).

http://creativecommons.org/licenses/by/4.0/

\begin{abstract}
Following the classical definition of factorization of matrix-functions, we introduce a definition of factorization for functional operators with involutive rotation on the unit circle. Partial indices are defined and their uniqueness is proven. In previous works, the main research method for the study scalar singular integral operators and Riemann boundary value problems with Carlemann shift were operator identities, which allowed to eliminate shift and to reduce scalar problems to matrix problems without shift. In this study, the operator identities were used for the opposite purpose: to transform operators of multiplication by matrix-functions into scalar operators with Carlemann linear-fractional shift.
\end{abstract}

\section{Keywords}

Carlemann Shift, Operator Identities, Factorization, Involutive Rotation

\section{Introduction}

A large number of works have been dedicated to Riemann boundary value problems and to the related singular integral equations. We point out some monographs that have already become classic on this subject [1] [2] [3] [4]. A special place is occupied by problems with shift in boundary conditions and equations with shift [5]. Listed monographs and their authors played a significant role in the development of this topic.

The problem of factorization of matrix functions is closely connected with the solution of matrix Riemann boundary value problems, for which effective solution methods have not yet been found [5] p. 24, Theorem 6. This explains the interest in and motivation for the study of this topic. 
In [6], we constructed operator identities with invertible operators, which transform a singular integral operator $A$ with involutive fractional linear shift into a vector singular integral operator $D$ without shift. Applications have been identified in which the main method of investigation was operator identities [7] [8] [9] [10].

Simplicity of the shift under consideration permits us, when studying the operator $A$, to avoid associated operators, and to avoid the appearance of compact operators and to obtain the operator identity, which directly connects the class of singular integral operators with shift and the class of matrix characteristic singular integral operators without shift. For an orientation-preserving shift, this corresponds to a similarity transform $\mathcal{F} A \mathcal{F}^{-1}=D$.

Moreover, it was noted that the operator identity transforms functional operators with shift and singular integral operators independently of each other, and the results of the transformations are not mixed. This makes it possible to directly apply the operator identity to operators with a shift and to transform them into the operator of multiplication by a matrix-function without the appearance of any shifts or singular operators.

The advantages of the proposed approach are manifested when considering various applications. In [8] [9], we proved invertibility conditions for singular integral operators with a direct shift and for matrix characteristic operators with coefficients having an automorphic structure generated by piecewise constant functions.

In [7], based on the known results on factorization [11], invertibility conditions in weighted Lebesgue spaces for singular integral operators with linear fractional involution and piecewise constant coefficients with three points of discontinuity were obtained. For homogeneous equations with such operators, the number of linearly independent solutions was calculated [10], the case when the coefficients of singular integral operators with four values has been considered and conditions for the non-triviality of the kernel of such operators were found.

Following this method, we studied a Riemann boundary value problem with a shift inward of the domain with piecewise constant coefficients taking two values [8] [9]. The conditions of existence and uniqueness of the homogeneous problem were found, as well as the formula for calculating the number of linearly independent solutions.

In this paper, we propose new applications of operator identities. We consider functional operators with involutive rotation on the unit circle. The main results are:

- A definition of factorization for these operators is given. The correctness of the definition is proven. Partial indices are defined and their uniqueness is proven.

- A relationship between the factorization of the matrix function and the factorization of the corresponding functional operator with shift is obtained. 


\section{Factorization of the Operators with Carlemann Rotation on the Unit Circle}

Let $T$ denote the unit circle. We review definitions that we are going to use [5] [12].

Factorization of non-degenerate matrix function $\boldsymbol{G}(t), \operatorname{det} \boldsymbol{G}(t) \neq 0$ in the space $L_{2}^{2}(T)$ is expressed by the representation

$$
\boldsymbol{G}(t)=\boldsymbol{G}^{+}(t) \Lambda(t) \boldsymbol{G}^{-}(t),
$$

were matrix functions $\boldsymbol{G}^{+}(t), \boldsymbol{G}^{-}(t)$ are the boundary values of analytic, non-degenerate within $D_{+}$and outside $D_{-}$of unit circle $T$, matrix functions $\boldsymbol{G}^{+}(z)$ and $\boldsymbol{G}^{-}(z), \operatorname{det} \boldsymbol{G}^{+}(z) \neq 0$ and $\operatorname{det} \boldsymbol{G}^{-}(z) \neq 0$, respectively;

$$
\Lambda(t)=\operatorname{diag}\left[t^{\kappa_{1}}, t^{\kappa_{2}}\right] \text {, }
$$

were $\kappa_{1}, \kappa_{2}$ are integers and $\kappa_{1} \geq \kappa_{2}$. The numbers $\kappa_{1}, \kappa_{2}$ are called partial indices.

It is known [5] [11] that the partial indices are invariants of the factorization and do not depend on a particular type of representation, and that the numbers $\kappa_{1}, \kappa_{2}$ are uniquely defined.

We use the following notations for projectors acting in space $L_{2}(T)$, $\mathcal{P}=\frac{1}{2}(I+W), \quad \mathcal{Q}=\frac{1}{2}\left(I_{T}-W\right)$ where $I$ is the identity operator, operator $W$ is the rotation operator:

$$
(W \varphi)(t)=\varphi(-t) .
$$

We introduce similar notation for identity operator $I$ in the space $L_{2}^{2}(T)$. The operation of square root extraction is denoted by $(N \varphi)(t)=\varphi(\sqrt{t})$.

Let us see how the factorization of a functional operator with shift looks if we proceed from the defition of the second-order matrix function factorization in the space $L_{2}^{2}(T)$. Let functions $a(t), b(t)$ be bounded measurable functions given on $T$. Factorization of an invertible operator

$$
A=a(t) I+b(t) W
$$

in the space $L_{2}(T)$ is expressed by its representation in the form

$$
\left[A^{+}(t) I+B^{+}(t) W\right] \Omega\left[A^{-}(t) I+B^{-}(t) W\right],
$$

were

$$
\Omega=\frac{1}{2}\left(t^{2 \kappa_{1}}+t^{2 \kappa_{2}}\right) I+\frac{1}{2}\left(t^{2 \kappa_{1}}-t^{2 \kappa_{2}}\right) W
$$

so that functions

$$
\begin{aligned}
& g_{11}^{ \pm}(t)=N \mathcal{P}\left(A^{ \pm}(t)+B^{ \pm}(t)\right), g_{12}^{ \pm}(t)=N\left[t \mathcal{Q}\left(A^{ \pm}(t)-B^{ \pm}(t)\right)\right], \\
& g_{21}^{ \pm}(t)=N\left[\frac{1}{t} \mathcal{Q}\left(A^{ \pm}(t)+B^{ \pm}(t)\right)\right], g_{22}^{ \pm}(t)=N \mathcal{P}\left(A^{ \pm}(t)-B^{ \pm}(t)\right),
\end{aligned}
$$

have to be boundary values of analytic non-singular functions

$g_{11}(z), g_{22}(z), g_{12}(z), g_{21}(z)$ in $D_{+}$and outside $D_{-}$of unit circle $T$ respec- 
tively, and the following inequalities have to be fulfilled $g_{11}(z) g_{22}(z) \neq g_{12}(z) g_{21}(z), z \in D_{+} \cup D_{-}$. We also provide other forms of representation (3), of operator $A$ and of (4) through the projectors

$$
\begin{gathered}
{\left[\left(A^{+}(t)+B^{+}(t)\right) \mathcal{P}+\left(A^{+}(t)-B^{+}(t)\right) \mathcal{Q}\right] \Omega\left[\left(A^{-}(t)+B^{-}(t)\right) \mathcal{P}+\left(A^{-}(t)-B^{-}(t)\right) \mathcal{Q}\right],} \\
A=(a+b) \mathcal{P}+(a-b) \mathcal{Q} \\
\Omega=t^{2 \kappa_{1}} \mathcal{P}+t^{2 \kappa_{2}} \mathcal{Q}
\end{gathered}
$$

We call integers $\kappa_{1}$ and $\kappa_{2}$ partial indices of $A$.

In works [6] [7] [9], a similarity transformation was constructed that transforms the identity operator $I$, acting in the space $L_{2}(T)$ to the identity operator $I$, acting in the space $L_{2}^{2}(T)$; the shift operator $W$, acting in space $L_{2}(T)$ to the matrix operator $V$, acting in the space $L_{2}^{2}(T)$ :

$$
\mathcal{F}^{-1} W \mathcal{F}=\boldsymbol{V}, \quad \boldsymbol{V}=\left[\begin{array}{cc}
1 & 0 \\
0 & -1
\end{array}\right]
$$

We also note that the operator of multiplication by a function transforms into

$$
\mathcal{F}^{-1} a(t) I \mathcal{F}=\left[\begin{array}{cc}
a(\sqrt{t})+a(-\sqrt{t}) & \sqrt{t}[a(\sqrt{t})-a(-\sqrt{t})] \\
\frac{1}{\sqrt{t}}[a(\sqrt{t})-a(-\sqrt{t})] & a(\sqrt{t})+a(-\sqrt{t})
\end{array}\right] .
$$

To describe the similarity transformation structure we need some definitions and operators.

Let $\Gamma$ and $\gamma$ be contours, and let $\gamma \subset \Gamma$. The extension of a function $f(t), t \in \gamma$, to $\Gamma \backslash \gamma$ by the value zero, will be denoted by $\left(J_{\Gamma \backslash \gamma} f\right)(t), t \in \Gamma$. The restriction of a function $\varphi(t), t \in \Gamma$ to $\gamma$ will be denoted by $\left(C_{\gamma} \varphi\right)(t)$, $t \in \gamma$.

The operator $\mathcal{F} \in\left[L_{2}^{2}(T), L_{2}(T)\right]$ is determined by the composition of the operators $M Z \Pi G N$.

In our case, these operators have the following form

$$
M_{T_{+}}\left[\begin{array}{l}
\psi_{1} \\
\psi_{2}
\end{array}\right]=J_{T_{-}} \psi_{1}+W J_{T_{-}} \varphi_{2}, \quad M_{T_{+}}^{-1} \varphi=\left[\begin{array}{c}
C_{T_{+}} \varphi \\
C_{T_{+}} W \varphi
\end{array}\right],
$$

where $T_{+}$and $T_{-}$are the upper and the lower parts of the unit circle, respectively,

$$
\begin{aligned}
& \boldsymbol{Z}^{ \pm 1}=\frac{1}{\sqrt{2}}\left[\begin{array}{rr}
1 & 1 \\
1 & -1
\end{array}\right], \Pi^{ \pm 1}=\operatorname{diag}\left[1, t^{ \pm 1}\right], N_{T_{+}} \zeta(t)=\zeta\left(t^{2}\right), N_{T_{+}}^{-1} \zeta(t)=\zeta\left(t^{\frac{1}{2}}\right), \\
& M_{T_{+}}^{-1} \in\left[L_{2}(T), L_{2}^{2}\left(T_{+}\right)\right], \quad M_{T_{+}} \in\left[L_{2}^{2}\left(T_{+}\right), L_{2}(T)\right], \quad N_{T_{+}} \in\left[L_{2}^{2}(T), L_{2}^{2}\left(T_{+}\right)\right] .
\end{aligned}
$$

\section{Uniqueness of Factorization and Partial Indices}

Theorem 1. The invertible operator $A$ in the space $L_{2}(T)$

$$
A=a_{T}(t) I+b_{T}(t) W
$$

admits factorization 


$$
A=\left[A^{+}(t) I+B^{+}(t) W\right] \Omega\left[A^{-}(t) I+B^{-}(t) W\right],
$$

if and only if the matrix function $\boldsymbol{G}(t)$ :

$$
\boldsymbol{G}(t)=\left[\begin{array}{cc}
\boldsymbol{N}_{T_{+}}^{-1} P_{W}\left(a_{T}(t)+b_{T}(t)\right) & \boldsymbol{N}_{T_{+}}^{-1}\left[t Q_{W}\left(a_{T}(t)-b_{T}(t)\right)\right] \\
\boldsymbol{N}_{T_{+}}^{-1}\left[\frac{1}{t} Q_{W}\left(a_{T}(t)+b_{T}(t)\right)\right] & \boldsymbol{N}_{T_{+}}^{-1} P_{W}\left(a_{T}(t)-b_{T}(t)\right)
\end{array}\right]
$$

admits factorization

$$
\boldsymbol{G}(t)=\boldsymbol{G}^{+}(t) \operatorname{diag}\left[t^{\kappa_{1}}, t^{\kappa_{2}}\right] \boldsymbol{G}^{-}(t)
$$

in the space $L_{2}^{2}(T)$.

Proof. We apply the operators $\mathcal{F}^{-1}$ and $\mathcal{F}$ on the left and right to the factorization (3) of the operator $A$ :

$$
\begin{aligned}
& a_{T}(t) I+b_{T}(t) W=\left[A^{+}(t) I+B^{+}(t) W\right] \Omega\left[A^{-}(t) I+B^{-}(t) W\right], \\
& \mathcal{F}^{-1}\left[a_{T}(t) I+b_{T}(t) W\right] \mathcal{F} \\
& =\mathcal{F}^{-1}\left[A^{+}(t) I+B^{+}(t) W\right] \mathcal{F F}^{-1} \Omega \mathcal{F} \mathcal{F}^{-1}\left[A^{-}(t) I+B^{-}(t) W\right] \mathcal{F} .
\end{aligned}
$$

We calculate

$$
\begin{aligned}
\mathcal{F}^{-1}\left[a_{T}(t) I+b_{T}(t) W\right] \mathcal{F} & \\
= & \frac{1}{2}\left[\begin{array}{cc}
a_{T}(\sqrt{t})+a_{T}(-\sqrt{t}) & \sqrt{t}\left[a_{T}(\sqrt{t})-a_{T}(-\sqrt{t})\right] \\
\frac{1}{\sqrt{t}}\left[a_{T}(\sqrt{t})-a_{T}(-\sqrt{t})\right] & a_{T}(\sqrt{t})+a_{T}(-\sqrt{t})
\end{array}\right] \boldsymbol{I} \\
& +\frac{1}{2}\left[\begin{array}{cc}
b_{T}(\sqrt{t})+b_{T}(-\sqrt{t}) & \sqrt{t}\left[b_{T}(\sqrt{t})-b_{T}(-\sqrt{t})\right] \\
\frac{1}{\sqrt{t}}\left[b_{T}(\sqrt{t})-b_{T}(-\sqrt{t})\right] & b_{T}(\sqrt{t})+b_{T}(-\sqrt{t})
\end{array}\right] \boldsymbol{V} .
\end{aligned}
$$

Then we calculate

$$
\mathcal{F}^{-1} \Omega \mathcal{F}=\mathcal{F}^{-1}\left[\frac{1}{2}\left[t^{2 \kappa_{1}}+t^{2 \kappa_{2}}\right] I+\frac{1}{2}\left[t^{2 \kappa_{1}}-t^{2 \kappa_{2}}\right] W\right] \mathcal{F}=\left[\begin{array}{cc}
t^{\kappa_{1}} & 0 \\
0 & t^{\kappa_{2}}
\end{array}\right] \boldsymbol{I} .
$$

and

$$
\begin{aligned}
\mathcal{F}^{-1}\left[A^{ \pm}(t) I_{T}+B^{ \pm}(t) W\right] \mathcal{F} & \\
= & \frac{1}{2}\left[\begin{array}{cc}
A^{ \pm}(\sqrt{t})+A^{ \pm}(-\sqrt{t}) & \sqrt{t}\left[A^{ \pm}(\sqrt{t})-A^{ \pm}(-\sqrt{t})\right] \\
\frac{1}{\sqrt{t}}\left[A^{ \pm}(\sqrt{t})-A^{ \pm}(-\sqrt{t})\right] & A^{ \pm}(\sqrt{t})+A^{ \pm}(-\sqrt{t})
\end{array}\right] \boldsymbol{I} \\
& +\frac{1}{2}\left[\begin{array}{cc}
B^{ \pm}(\sqrt{t})+B^{ \pm}(-\sqrt{t}) & \sqrt{t}\left[B^{ \pm}(\sqrt{t})-B^{ \pm}(-\sqrt{t})\right] \\
\frac{1}{\sqrt{t}}\left[B^{ \pm}(\sqrt{t})-B^{ \pm}(-\sqrt{t})\right] & B^{ \pm}(\sqrt{t})+B^{ \pm}(-\sqrt{t})
\end{array}\right] \boldsymbol{V} .
\end{aligned}
$$

Formulas (7) describe the relationships between the factors of the operator $A$ and the matrix $\boldsymbol{G}(t)$. The matrix from (7) coincides with the matrix $\boldsymbol{G}^{ \pm}(t)=\left[\begin{array}{ll}g_{11}^{ \pm}(t) & g_{12}^{ \pm}(t) \\ g_{21}^{ \pm}(t) & g_{22}^{ \pm}(t)\end{array}\right]$, composed of functions included in the definition of 
factorization of a functional operator. Taking into account the requirements that are imposed on the functions $g_{11}^{ \pm}(t), g_{22}^{ \pm}(t), g_{12}^{ \pm}(t), g_{21}^{ \pm}(t)$ in the definition of factorization of functional operator with shift $A$, we come to the classical definition of factorization (1), (2) of a second-order matrix-valued function $\boldsymbol{G}(t)$ in the space $L_{2}^{2}(T)$.

From Theorem 2, it follows that the partial indices $k_{1}, k_{2}$ in our representation (3), (4) are uniquely determined, because they are uniquely determined by the classical factorization of the matrix $\boldsymbol{G}(t)$.

Corollary 1. Partial indeces $\kappa_{1}$ and $\kappa_{2}$ of the invertible functional operator

$$
A=a_{T}(t) I+b_{T}(t) W
$$

in the space $L_{2}(T)$ are uniquely determined and do not depend on the specific realization of factorization (3), (4) and coincide with the partial indices of the matrix function $\boldsymbol{G}(t)$.

\section{Conclusion}

This paper presents the concept of functional factorization operator with Carlemann rotation on the unit circle. The main method of investigation was operator identities. Operator identities present a convenient mathematical tool for studying Riemann boundary value problems, singular integral equations and with involutive fractional rational shift and factorization problems.

\section{Conflicts of Interest}

The authors declare no conflicts of interest regarding the publication of this paper.

\section{References}

[1] Gakhov, F.D. (1977) Boundary Value Problems, Mathematics in Science and Engineering. Nauka, Moscow.

[2] Muskhelishvili, N.I. (2008) Singular Integral Equations, Boundary Value Problems of the Theory of Functions and Some of Their Applications to Mathematical Phisics. Dover Publications, Mineola.

[3] Gohberg, I. and Krupnik, N. (1992) One-Dimensional Linear Singular Integral Equations. In: Operator Theory: Advances and Applications, Birkhauser Verlag, Basel, Boston, Berlin, Vol. 54. https://doi.org/10.1007/978-3-0348-8602-4

[4] Mikhlin, S.G. and Prossdorf, S. (1986) Singular Integral Operators. Akademie-Verlag, Berlin.

[5] Litvinchuk, G.S. (2000) Solvability Theory of Boundary Value Problems and Singular Integral Equations with Shift. Kluwer Academic Publishers, Dordrecht, Boston, London. https://doi.org/10.1007/978-94-011-4363-9

[6] Karelin, A.A. (2001) On a Relation between Singular Integral Operators with a Carlemann Linear-Fractional Shift and Matrix Characteristic Operators without Shift. Boletín de la Sociedad Matemátlca Mexicana, 7, 235-246.

[7] Karelin, A.A. (2002) On the Operator Equality and Some of Its Application. Proceedings of A. Razmadze Mathematical Institute, 128, 105-116. 
[8] Karelin, A.A., Perez-Lechuga, G. and Tarasenko, A.A. (2008) Riemann Problem and Singular Integral Equations with Coefficients Generated by Piecewise Constant Functions. Differential Equations, 44, 1225-1235.

https://doi.org/10.1134/S0012266108090048

[9] Karelin, A. (2007) Applications of Operator Equalities to Singular Integral Operators and to Riemann Boundary Value Problems. Mathematische Nachrichten, 280, 1108-1117. https://doi.org/10.1002/mana.200510539

[10] Karelin, A. (2008) Singular Integral Operators with Coefficients of a Special Structure Related to Operator Equalities. Complex Analysis and Operator Theory, 2, 549-567. https://doi.org/10.1007/s11785-008-0066-X

[11] Spitkovski, I.M. and Tashbaev, A.M. (1997) Factorization of Certain Piecewise Constant Matrix Functions and Its Application. Mathematische Nachrichten, 151, 241-261. https://doi.org/10.1002/mana.19911510115

[12] Litvinchuk, G.S. and Spitkovski, I.M. (1987) Factorization of Measurable Matrix Functions. In: Operator Theory: Advances and Aplication, Birkhauser Verlag, Basel, Boston, Vol. 25. https://doi.org/10.1007/978-3-0348-6266-0 